\title{
The Warwick Hip Trauma Evaluation - an abridged protocol for the WHiTE Study
}

\author{
A MULTIPLE EMBEDDED RANDOMISED CONTROLLED TRIAL COHORT \\ STUDY
}

\section{L. Griffin, J. Achten, N. Parsons, F. Boardman, F. Griffiths, M. L. Costa}

\section{From Warwick} Medical School, University of Warwick, Coventry, United Kingdom

X. L. Griffin, MA, MSc, MRCS, NIHR Academic Clinical Fellow E J. Achten, PhD, Research Manager

Warwick Orthopaedics, Warwick Medical School, University of Warwick, Clifford Bridge Road, Coventry CV2 2DX, UK.

N. Narsons, PhD, Medical Statistician

- F. Boardman, PhD, Research Fellow

E. Griffiths, PhD, FRCGP, Professor of Medicine in Society Warwick Medical School, University of Warwick, Gibbet Hill Road, Coventry CV4 7AL, UK.

M. L. Costa, PhD, FRCS(Tr \& Orth), Professor of Trauma and Orthopaedic Surgery

Clinical Trials Unit, Warwick Medical School, University of Warwick, Gibbet Hill Road, Coventry CV4 7AL, UK.

Correspondence should be sent to $\mathrm{Mr}$ X. L. Griffin; e-mail: x.griffin@warwick.ac.uk

10.1302/2046-3758.111 $2000127 \$ 2.00$

Bone Joint Res 2012;1:310-14. Received 15 August 2012; Accepted after revision 23 October 2012

Fractures of the proximal femur are one of the greatest challenges facing the medical community, constituting a heavy socioeconomic burden worldwide. The National Hip Fracture Audit currently provides a framework for service evaluation. This evaluation is based upon the assessment of process rather than assessment of patient-centred outcome and therefore it fails to provide meaningful data regarding the clinical effectiveness of treatments. This study aims to capture data from the cohort of patients who present with a fracture of the proximal femur at a single United Kingdom Major Trauma Centre. Patientcentred outcomes will be recorded and provide a baseline cohort within which to test the clinical effectiveness of experimental interventions.

Keywords: Hip fractures, Cohort studies, Controlled clinical trial, Outcome assessment (Health Care), Interview, Frail older adults

\section{Introduction}

Fractures of the proximal femur are one of the greatest challenges facing the medical community. In 1990, a global incidence of 1.31 million was reported and was associated with 740000 deaths. ${ }^{1}$ Proximal femoral fractures constitute a heavy socioeconomic burden worldwide. The cost of this clinical problem is estimated at 1.75 million disability adjusted life years lost: $1.4 \%$ of the total healthcare burden in established market economies. ${ }^{1}$ Acute United Kingdom NHS Trusts are required to follow-up and record data about all patients admitted with a fracture of the proximal femur as part of the National Hip Fracture Database (NHFD) Audit. $^{2}$ Currently, this audit provides a framework for service evaluation. This evaluation is based upon the assessment of process rather than assessment of patient-centred outcome, and therefore it fails to provide meaningful data regarding the clinical effectiveness of treatments. This study aims to capture data from the cohort of patients who present with a fracture of the proximal femur at the University Hospitals Coventry and Warwickshire NHS Trust, one of 22 Major Trauma Centres in England. We will record important patient-centred outcomes and provide a baseline cohort within which to test the clinical effectiveness of alternative interventions.

\section{Materials and Methods}

Study design. This will be a cohort study that will facilitate multiple embedded randomised controlled trials as described by Relton et al. ${ }^{3}$ The study will be performed at a single Major Trauma Centre (University Hospitals Coventry and Warwickshire NHS Trust) in the United Kingdom.

Ethical approval. This study has been reviewed by the London - Camberwell St Giles Research Ethics Committee (Ref: 11/LO/0927). The study was given ethical approval on 18 August 2011. The research will be carried out in compliance with the Helsinki Declaration.

Study registration. This study has been registered with the International Standard Randomised Controlled Trial Number Register (ISRCTN 63982700) and the NIHR CRN (Comprehensive Research Network) Portfolio (UKCRN ID 12351).

Study participants. All patients aged $\geq 60$ years with a fracture of the proximal femur, including those with cognitive impairment, are eligible for inclusion in this study. This broad eligibility criterion should ensure that the results of the study can be readily generalised to the wider population of patients with fracture of the proximal femur. Age of 60 years is commonly used as a surrogate marker of bone density; i.e. patients aged $>60$ years represent those with a fragility fracture. Patients will be excluded if they are deemed by the Consultant Trauma 
Surgeon to be medically unfit for an operation and are treated non-operatively.

Recruitment. Patients will be recruited from trauma meetings at University Hospitals Coventry and Warwickshire NHS Trust. Once an eligible patient is identified by the responsible Consultant Trauma Surgeon they will be referred to a research associate.

Timeline. The study commenced recruitment on 17 January 2012. Recruitment is planned to last for one year in the first instance. Interim analyses as described in the statistical analysis will be reported as appropriate.

Consent. The large majority of patients with fracture of the proximal femur are a clinical priority for urgent operative care. They will undergo surgery on the day of, or the day following, admission. All patients with a fracture of the proximal femur are in pain and many have received opiate analgesia. It is therefore understandable that patients often find the initial period of their treatment in hospital confusing and disorientating. Similarly, patients' next of kin, carers and friends are anxious at this time and may also have difficulty in weighing the large amounts of information that they are given about the injury and plan for treatment.

In this emergency situation the focus lies with obtaining consent for surgery (where possible) and informing the patient and any next of kin about the immediate clinical care. There is limited time for the patient, or if they lack capacity their consultee, to review trial documentation and make an informed decision about whether they would wish to participate.

Conducting research in this 'emergency setting' is regulated by the Mental Capacity Act $2005 .{ }^{4}$ As patients are likely to lack capacity as described above, and because of the urgent nature of the treatment limiting access to, and appropriate discussion with personal consultees, we will act in accordance with section 32, subsection $9 b$ of the Mental Capacity Act. We will not obtain consent before surgery but inform the patient and seek patient consent, or consultee agreement, for continuation in the study at the first appropriate time point in the post-operative period.

At the first appropriate time when the patient has regained capacity (this will usually be on the first day after surgery) the research associate will provide the participant with all of the study information. The participant will be given the opportunity to ask questions and discuss the study with their family and carers. They will then be asked to provide written consent for follow-up within the study.

For participants who do not regain capacity or lack capacity, as a result of pre-fracture chronic cognitive impairment, reasonable efforts will be made to identify a Personal Consultee as described in the Mental Capacity Act 2005. If no Personal Consultee can be identified then a Nominated Consultee will be identified to advise the research team. At all times the Chief Investigator will act in accordance with the participants' best interests.
Any new information that arises during the trial that may affect participants' willingness to take part will be reviewed by the Programme Management Group; if necessary this will be communicated to all participants. A revised consent form will be completed if necessary.

Post-recruitment withdrawals and exclusions. Participants may withdraw from the study at any time without prejudice. The General Practitioners of those participants who are 'lost to follow-up' will be contacted in order to attempt to complete the follow-up. Participants may be withdrawn from the study at the discretion of the Chief Investigator due to safety concerns.

Study interventions. The standard-of-care treatment for participants entering the cohort study will follow the usual practice and pathways at the University Hospitals Coventry and Warwickshire NHS Trust. This will be as follows:

Pre-operative assessment. Participants will usually be assessed in the Emergency Department. Diagnosis of a fracture of the proximal femur will be confirmed by a plain anteroposterior (AP) radiograph of the pelvis. Where there is doubt over the radiological diagnosis, an additional plain lateral hip radiograph may be taken. Where diagnostic uncertainty remains, participants will be reviewed by the on-call Orthopaedic Surgeon, and where clinically indicated, a CT or MRI of the pelvis will be performed. For participants who fall and sustain an injury to the proximal femur while in hospital, a similar diagnostic protocol will be followed, except that the responsible clinician will conduct the initial assessment on the inpatient ward.

Participants will be transferred to an Orthopaedic Trauma ward. All participants will undergo the following investigations as a minimum: electrocardiogram, full blood count, blood group and save, coagulation screen, urea, creatinine and electrolytes. In accordance with Trust guidelines, participants with haemoglobin less than $9 \mathrm{~g} / \mathrm{dl}$, or between $9 \mathrm{~g} / \mathrm{dl}$ and $9.9 \mathrm{~g} / \mathrm{dl}$ and a history of ischaemic heart disease (IHD), will be transfused to at least $10 \mathrm{~g} / \mathrm{dl}$; between $9 \mathrm{~g} / \mathrm{dl}$ and $9.9 \mathrm{~g} / \mathrm{dl}$ and no history of IHD will have two units of packed red cells cross-matched and available intra-operatively. All participants undergoing a blood product transfusion will have a full blood count post-transfusion.

All patients will be assessed for venothromboembolic risk. Where appropriate, chemo- and mechanical prophylaxis will be prescribed as per Trust guidelines. In participants with an international normalised ratio (INR) greater than 6.5 , advice will be sought immediately from the oncall haematologist. Participants with an INR between 2 and 6.5 will receive vitamin K $1 \mathrm{mg}$ intravenously (IV). Participants at low risk of complications of reversal (atrial fibrillation, recurrent thromboembolic event, thromboembolic event more three months previously or thrombophilia) will receive routine thromboprophylaxis after four hours. Those at high risk (prosthetic heart valve, caval filter or thromboembolic event less than three months previously) will receive heparin via a standard IV infusion. Each 
participant will have their INR checked at 7 am on the day of surgery. Participants with an INR greater than 2.5 will be delayed for 24 hours and have their INR checked again; an INR between 1.5 and 2.5 will receive two units of fresh frozen plasma immediately pre-operatively; an INR less than 1.5 will undergo routine surgery.

Analgesia will be started from admission as $1 \mathrm{~g}$ paracetamol oral or IV qds and $30 \mathrm{mg}$ codiene phosphate oral qds. These will continue during the nil-by-mouth period. The participants' regular anti-hypertensive, antireflux (omeprazole, rantidine etc), anti-anginal and bronchodilator therapies will be continued during the nilby-mouth period.

Anaesthetic technique. A regional or general anaesthesia technique will be used for every participant at the discretion of the senior attending anaesthetist. Intraoperative analgesia will be achieved by combining a local anaesthetic nerve block (femoral and lateral cutaneous nerve of the thigh, fascia iliaca or lumbar plexus) using either a nerve stimulator or ultrasound-guided technique, IV paracetamol $1 \mathrm{~g}$ intravenous infusion and opiate analgesia as clinically indicated.

Operative intervention. Antibiotic prophylaxis will be prescribed in accordance with Trust guidelines; participants who can tolerate penicillins will receive $1 \mathrm{~g}$ flucloxacillin and 3 to $5 \mathrm{mg} / \mathrm{kg}$ gentamicin at induction as an IV infusion over 15 to 30 minutes. Penicillin-sensitive participants will receive teicoplanin $600 \mathrm{mg}$, or $800 \mathrm{mg}$ if body mass is $>80 \mathrm{~kg}$, as an IV bolus and 3 to $5 \mathrm{mg} / \mathrm{kg}$ gentamicin as an IV infusion over 15 to 30 minutes. Those who have a positive methicillin-resistant Staphylococcus aureus (MRSA) screen will be given the same prophylaxis as those who are penicillin-sensitive.

Fractures will be classified pre-operatively from the plain AP pelvis radiograph into four groups: 1) Minimally displaced intracapsular - proximal to the trochanteric line/crest ${ }^{5-7}$; 2) Displaced intracapsular - proximal to the trochanteric line/crest ${ }^{5-7}$; 3) Trochanteric - distal to the trochanteric line/crest and proximal to the lesser trochanter $^{5}$; and 4) Subtrochanteric - fractures located principally in the $5 \mathrm{~cm}$ below the lesser trochanter. ${ }^{5}$

Group 1: Minimally displaced intracapsular fractures. All participants will have their fracture fixed in situ. The lower limb will be supported on a fracture table. Internal fixation of the fracture will be achieved through a standard mini-open lateral or percutaneous approach with two or three parallel cannulated screws.

Group 2: Displaced intracapsular fractures. Participants will be positioned in the lateral position. The operating surgeon will perform their preferred approach. The hip will be dislocated and the head excised. A standard polished, tapered, modular head hemiarthroplasty femoral component will be cemented into the femur using a third generation cementing technique. The femoral head will be applied and the arthroplasty reduced into the native acetabulum.
A subgroup of these participants may be offered total hip replacement (THR) where the acetabulum is also replaced. Given the lack of evidence to support criteria to select those participants who might benefit from THR, ${ }^{8}$ a pragmatic approach will be adopted. The responsible Consultant Trauma Surgeon will determine eligibility for this subgroup, on the basis that he or she believes the participant would benefit from THR. In the event of a participant undergoing THR, the approach, implant and operative technique employed would be at the discretion of the operating surgeon.

Group 3: Trochanteric fractures. All participants will have a closed reduction of their fracture. The lower limb will be supported on a fracture table. Internal fixation of the fracture will be achieved through a standard lateral approach with a dynamic hip screw.

Group 4: Subtrochanteric fractures. All participants will have a closed reduction of their fracture. The lower limb will be supported on a fracture table. Internal fixation of the fracture will be achieved through a standard lateral, mini-open or percutaneous approach for nailing. Fixation will be achieved using a long, distally locked, cephalomedullary device.

Post-operative rehabilitation. Post-operative analgesia will be prescribed intra-operatively and reviewed by the responsible clinical teams as appropriate. The initial postoperative regimen will be: paracetamol $1 \mathrm{~g}$ oral or IV qds, codeine phosphate $30 \mathrm{mg}$ oral or IM for two days, lactulose $20 \mathrm{mg}$ oral bd, oramorph $10 \mathrm{mg}$ every two hours prn and buccal prochlorperazine $6 \mathrm{mg}$ bd prn. In addition oxygen 2l/min will be prescribed for two days. Prescribed chemothromboprophylaxis will be continued for a period of between 28 and 35 days.

Between 24 and 48 hours in the post-operative period participants will undergo an initial Physiotherapy and Occupational Therapy trauma assessment. A full social, cognitive, premorbid function and falls history will be obtained and documented. Participants will be given the relevant University Hospitals Coventry and Warwickshire NHS Trust Patient Information packs. An initial treatment plan with objectives will be made, recorded and commenced. The aim of this plan will be for participants to mobilise through early and active full-weight-bearing.

All participants will have a clinical review by a Specialist Orthogeriatrician within 72 hours of admission. This will include a fracture prevention assessment. Assessment and treatment of participants' for osteoporosis will be carried out in accordance with current National Institute of Health and Clinical Excellence (NICE) guidance, ${ }^{9}$ similarly participants' risk of falling in accordance with British Orthopaedic Association (BOA) guidance. ${ }^{10}$

Social support and discharge requirements will be identified as soon as possible. Identification and completion of relevant referrals will be made within seven days as appropriate. Additionally, appropriate referrals to other agencies or departments will be made to initiate or 
complete a falls prevention plan. Participants will be discharged from the acute Orthopaedic Trauma Ward at the earliest safe opportunity to the most appropriate discharge destination as determined by the multidisciplinary team. Final discharge from NHS facilities will be at the earliest safe opportunity.

Follow-up. Following consent, participants will have their pre-operative hip function, quality of life and physical activity level assessed through questionnaires (EuroQol (EQ-5D), ${ }^{11}$ ICECAP(O) ${ }^{12-15}$ and Oxford hip score ${ }^{16}$ ). This will be followed by telephone questionnaires at four weeks, and four and 12 months post-operatively. For those participants with cognitive impairment this assessment will be limited to the EQ-5D, which will be reported by an appropriate proxy. Participants and their consultees will also be invited through a separate information sheet and consent form to take part in semi-structured interviews at these time points. Where particpants have died and the research team is aware (and the event already recorded as an adverse event) no further follow-up is attempted. If the research team is unaware then contact will be made - for example out-of-area patients - and the event of death recorded and further follow-up ceased.

In this study we will use techniques common in longterm cohort studies to ensure minimum loss to follow-up. Multiple contact addresses and telephone numbers, mobile numbers and email addresses will be recorded during enrolment. Considerable effort will be made by the research team to keep in communication with participants.

We will attempt to contact the participant or next of kin by telephone on three occasions, and then send them a postal questionnaire invitation letter and questionnaire to complete with a pre-paid envelope. Finally we will confirm contact details with the participant's General Practitioner. If all these methods fail then we will class the participant as a non-responder for that time point.

Semi-structured interviews. Participants, both those with and without cognitive impairment, and their carers will be recruited for semi-structured interviews at each follow-up time point (within one month, four months, one year postoperatively). Participants and carers who have consented to participate in a semi-structured interview on entry to the study will be contacted by the project researcher to arrange an interview. After completion of 15 to 20 interviews, the team will review the EQ-5D scores and time points at interview of the participants. Further purposeful sampling will ensure, as far as is possible, that our final sample will include a range of time points and EQ-5D scores. This process will enable us to identify differences in the expectations and priorities of participants across different time points in their recovery and for participants of differing functioning and quality-of-life before their fracture. This method of purposeful sampling will be continued (with further review after approximately 30 interviews) until approximately 45 interviews have been completed. Interviewing will continue until data saturation has occurred.
Sample size. No formal sample size calculation has been conducted for the comprehensive cohort study. However, based upon data from the NHFD, between 500 and 600 patients with a fracture of the proximal femur can be expected to be treated at University Hospitals Coventry and Warwickshire NHS Trust each year. This will be an ongoing study during which interim analyses will be performed as appropriate.

Qualitative analysis. The interviews with participants and their carers will be audio recorded, transcribed verbatim and analysed with the assistance of Nvivo 8 qualitative data analysis software (QSR International Ltd, Melbourne, Australia). Thematic analysis of interviews will focus on participant expectations and priorities. Interview participants' scores on the measurement tools, as well as their thoughts and reflections on the ease of use of the tool will be analysed in line with their interview transcript. In particular, the team will focus on whether the themes identified from the interviews are adequately captured by the measurement tools, and crucially, whether there are themes that are missed by the tool but are nevertheless important to patients experiencing fracture of the proximal femur. Thus, interview participants' scores on the measurement tools will be treated as part of the qualitative data.

\section{Reporting plan}

This study is expected to report its initial findings in March 2013.

\section{References}

1. Johnell $\mathbf{0}$, Kanis JA. An estimate of the worldwide prevalence, mortality and disability associated with hip fracture. Osteoporosis Int 2004;15:897-902

2. National Hip Fracture Database. www.nhfd.co.uk (date last accessed 6 November 2012).

3. Relton C, Torgerson D, O'Cathain A, Nicholl J. Rethinking pragmatic randomised controlled trials: introducing the "cohort multiple randomised controlled trial" design. BMJ 2010;340:1066

4. No authors listed. Mental Capacity Act 2005. www.legislation.gov.uk/ukpga/ 2005/9/contents (date last accessed 6 November 2012).

5. Muller ME. Appendix A: The comprehensive classification of fractures of long bones. In: Muller ME, Allgower A, Schneider R, Wiilenegger H, eds. Manual of internal fixation: techniques recommended by the AO-ASIF Group. Third ed. Berlin Heidelberg New York: Springer-Verlag, 1995:118-150.

6. Parker MJ. Garden grading of intracapsular fractures: meaningful or misleading? Injury 1993;24:241-242.

7. Garden RS. Low-angle fixation in fractures of the femoral neck. $J$ Bone Joint Surg [Br] 1961;43-B:647-663

8. Carroll C, Stevenson M, Scope A, Evans P, Buckley S. Hemiarthroplasty and total hip arthroplasty for treating primary intracapsular fracture of the hip: a systematic review and cost-effectiveness analysis. Health Technol Assess 2011:15:1-74

9. National Institute for Health and Clinical Excellence (NICE). TA161 Osteoporosis: secondary prevention including strontium ranelate, 2011. Alendronate, etidronate, risedronate, raloxifene, strontium ranelate and teriparatide for the secondary prevention of osteoporotic fragility fractures in postmenopausal women. http://www.nice.org.uk/TA161 (date last accessed 21 September 2012).

10. Marsh D, Currie C, Brown P, et al. The care of elderly patients with fragility fracture. London: British Orthopaedic Association, 2007.

11. No authors listed. Euro0oL EQ-5D. http://www.euroqol.org/ (date last accessed 21 September 2012 
12. No authors listed. ICEpop CAPability measure for Older people (ICECAP-0) http://www.icecap.bham.ac.uk/index.shtml (date last accessed 21 September 2012).

13. Coast J, Flynn TN, Natarajan L, et al. Valuing the ICECAP capability index for older people. Soc Sci Med 2008;67:874-882.

14. Coast J, Peters TJ, Natarajan L, Sproston K, Flynn T. An assessment of the construct validity of the descriptive system for the ICECAP capability measure for older people. Qual Life Res 2008;17:967-976.

15. Grewal I, Lewis J, Flynn T, et al. Developing attributes for a generic quality of life measure for older people: preferences or capabilities? Soc Sci Med 2006;62:1891-1901

16. Dawson J, Fitzpatrick R, Carr A, Murray D. Questionnaire on the perceptions of patients about total hip replacement. J Bone Joint Surg [Br] 1996;78-B:185190.
Funding statement:

- Funding for this study has been received in the form of a National Institute for Health Research (NIHR) Programme Development Grant (UK) Ref: RP-DG-1210-10022

Author contributions:

- X. L. Griffin: Study design, Drafting manuscript, Principal investigator

I. Achten: Study design, Critical revision

- N. Parsons: Study design, Drafting manuscript, Study statistician

- F. Boardman: Study design

- F. Griffiths: Study design

- M. L. Costa: Study design, Critical revision, Chief investigator

ICMJE Conflict of Interest:

- None declared

(c)2012 British Editorial Society of Bone and Joint Surgery. This is an open-access article distributed under the terms of the Creative Commons Attributions licence, which permits unrestricted use, distribution, and reproduction in any medium, but not for commercial gain, provided the original author and source are credited. 